\title{
6 Forschungsfragen
}

Aus der gerade vorgetragenen Kritik an der Forschung zur dynamischen Routine ergibt sich die Notwendigkeit einer systematischen Auseinandersetzung mit der Frage der Routineninterdependenz. Erst wenn klar ist, wie in Organisationen mit Interdependenzen zwischen Routinen umgegangen werden kann und welche Konsequenzen sich daraus für die Entwicklung der Organisation ergeben, wird die sich im blinden Fleck der aktuellen Forschung zur dynamischen Routine befindliche Fragestellung nach den sich aus dem Bestandsproblem der Organisation ergebenden Herausforderungen des organisationalen Designs wieder widerspruchsfrei thematisierbar. Dementsprechend nähere ich mich im nun folgenden Kapitel zuerst auf rein konzeptuellem Wege einer Antwort auf die zwei zentralen Forschungsfragen dieser Arbeit:

1. Wie lässt sich die Form von Interdependenzbeziehungen zwischen Routinen konzeptualisieren?

2. Welche Dynamiken ergeben sich aus Interdependenzbeziehungen zwischen Routinen? 